\title{
A TEST FOR AN ABRUPT CHANGE IN WEIBULL HAZARD FUNCTIONS WITH STAGGERED ENTRY AND TYPE I CENSORING
}

\author{
Matthew R. Williams and Dong-Yun Kim \\ Department of Statistics, Virginia Tech, Blacksburg, VA 24061-0439
}

\begin{abstract}
We consider a test of an unknown change-point in a Weibull hazard function. We assume that data are subject to staggered entry and type I censoring. We formulate the profile log-likelihood ratio test statistic as a function of the changepoint and derive the limiting Gaussian process. From the supremum of the limiting process, we determine critical values and study the power of the test through simulation. We demonstrate this method using real data from a clinical study for the treatment of chronic granulomatous disease.

Key words and phrases: Change-point, Gaussian process, hazard function, Likelihood ratio test, Rayleigh distribution, staggered entry, type I censoring.
\end{abstract}

1. Introduction: An abrupt change in a hazard function often symbolizes the crossing of a threshold. One example occurs in clinical studies when a lag exists between the time when a treatment is administered and the time when symptoms have improved. Similarly, rates of mortality or cancer recurrence might change abruptly after a delay following the treatment. It may be useful to test for the existence of such changes before attempting to estimate them. Matthews and Farrell $(1982,1985)$ considered the case of a constant hazard rate with an abrupt change at an unknown time. They developed a likelihood ratio test for such a change-point and simulated critical values. Matthews, Farrell and Pyke (1985) demonstrated the weak convergence of a score test to a Brownian Bridge and derived asymptotic critical values for the test. Worsley (1988) noted that the likelihood ratio test was sensitive to the width of the data intervals. For example, if the unknown change-point of interest is allowed to be at the largest or smallest observation then the likelihood ratio test becomes unbounded. Davies (1977) considered hypothesis testing in more general terms in which nuisance parameters exist only under the alternative. Davies concluded that the standard likelihood ratio test asymptotics are not applicable, but inference from a Gaussian 
process may be possible. Loader (1991) also considered a constant hazard rate with abrupt change and used large deviation approximations to the significance level of the likelihood ratio test. Loader demonstrates the method on heart transplant data.

Recent works have expanded the scope of change-points within the constant hazard rate framework. Lu, Turnbull and Clark (1997) developed a likelihood ratio test including binary covariates, testing the null hypothesis of a change-point at $\nu=\nu_{0}$ vs. the alternative $\nu \neq \nu_{0}$. Dupuy (2009) allowed for the regression coefficients of the covariates to change as well, and obtained non-asymptotic bounds for the significance level and type II error for data with random right censoring. Goodman, Li and Tiwari (2006) test for multiple change-points in piecewise linear hazard functions using asymptotic Wald tests. Qin and Sun (1997) examine right-censored data with the hazard function completely specified before the change, but completely unknown after it. They propose a modified maximal censored likelihood ratio test and bootstrap to get critical values.

Kim, Woodroofe and $\mathrm{Wu}$ (2004) developed a likelihood ratio test for a change in hazard rates under staggered entry and type I censoring. Their test statistic converges weakly to a stationary Guassian process, whose tail probabilities can be approximated. We demonstrate that similar techniques can be employed to derive a test for data that has a Weibull distribution with known shape parameter under the null hypothesis. A known shape parameter corresponds to a known polynomial shape for the hazard function and allows for closed forms for maximum likelihood estimates of the scale parameter.

We consider a general form for the hazard function with unknown change point $\nu$ :

$$
h(t)=\left\{\begin{array}{ll}
\theta_{1} t^{k-1}, & t<\nu \\
\theta_{2} t^{k-1}, & t \geq \nu
\end{array}\right\}
$$

where $t \geq 0$ is the failure time, $k>0$ is the known shape parameter, and $\theta_{1}>0$ and $\theta_{2}>0$ are the scale parameters. The corresponding density is

$$
f(t)=\left\{\begin{aligned}
\theta_{1} t^{k-1} \times \exp \left(-\frac{\theta_{1}}{k} t^{k}\right), & t<\nu \\
\theta_{2} t^{k-1} \times \exp \left(-\frac{\theta_{1}}{k} \nu^{k}-\frac{\theta_{2}}{k}\left(t^{k}-\nu^{k}\right)\right), & t \geq \nu
\end{aligned}\right\}
$$

Note that $k=1$ is the exponential case. 
We consider the case of staggered entry and type I censoring. Subjects enter the study randomly, following a Poisson process. The ending time of the study $T$ is independent of the number of subjects or events. Conditioning on the total number of subjects, $N(T)=n$, who entered the study before time $T$, the censoring time of each subject is a sample from a uniform distribution, $\left(T-\tau_{i}\right) \sim U(0, T)$, where $\tau_{i}$ is the time the $i^{t h}$ subject entered the study. For simplicity we assume $\tau_{1}<\ldots<\tau_{n}$.

2. Main Result: Under the null hypothesis, there is no change, $\theta_{1} \neq \theta_{2}$. This formulation cancels out the change point, $\nu$, in the density (1.2), simplifying to a parameterization of a Weibull distribution with $k>0$ known. Under the null hypothesis, the failure time of a subject, $Y$, has a density:

$$
f(y)=\theta y^{k-1} \times \exp \left(-\theta y^{k} / k\right), y>0 .
$$

Under the alternative hypothesis, $\theta_{1} \neq \theta_{2}$, we use (1.2).

If we allow for staggered entry at time $\tau>0$ and right censoring at time $T$, our observed data is $X=\min (Y, T-\tau)$ and $\delta=1_{\{Y<T-\tau\}}$. Then for $N(T)=n$ independent subjects, the log-likelihood becomes

$\ln L_{N}\left(\theta_{1}, \theta_{2}, \nu\right)=K_{1}(\nu) \ln \left(\theta_{1}\right)+K_{2}(\nu) \ln \left(\theta_{2}\right)-T_{1}(\nu) \theta_{1}-T_{2}(\nu) \theta_{2}+\sum_{i=1}^{n} \delta_{i}(k-1) \ln x_{i}$,

where

$$
\begin{aligned}
K_{1}(\nu) & =\sum_{i=1}^{n} 1_{\left\{x_{i}<\nu, \delta_{i}=1\right\}} \\
K_{2}(\nu) & =\sum_{i=1}^{n} 1_{\left\{x_{i} \geq \nu, \delta_{i}=1\right\}} \\
T_{1}(\nu) & =\frac{1}{k} \sum_{i=1}^{n}\left(x^{k} \wedge \nu^{k}\right) \\
T_{2}(\nu) & =\frac{1}{k} \sum_{i=1}^{n}\left(x^{k}-\nu^{k}\right)_{+},
\end{aligned}
$$

with $(x \wedge \nu)=\min (x, \nu)$ and $(x-\nu)_{+}=\max (0,(x-\nu))$.

Under the null hypothesis, the maximum likelihood estimate (MLE) for $\theta$ is $\hat{\theta}=\left(K_{1}+K_{2}\right) /\left(T_{1}+T_{2}\right)$. Note that $\hat{\theta}$ is not a function of $\nu$. Under the alternative hypothesis, the conditional MLE's are $\hat{\theta_{1}}(\nu)=K_{1}(\nu) / T_{1}(\nu)$ and $\hat{\theta_{2}}(\nu)=K_{2}(\nu) / T_{2}(\nu)$, which depend on $\nu$. The log-likelihood ratio test statistic is the process 


$$
\Lambda_{N}(\nu)=K_{1}(\nu) \ln \left(\frac{K_{1}(\nu)}{T_{1}(\nu)} \times \frac{T_{1}+T_{2}}{K_{1}+K_{2}}\right)+K_{2}(\nu) \ln \left(\frac{K_{2}(\nu)}{T_{2}(\nu)} \times \frac{T_{1}+T_{2}}{K_{1}+K_{2}}\right) .
$$

We restrict $0<a \leq \nu \leq b<T$ for arbitrary $a$ and $b$ to ensure that $\Lambda_{N}(\nu)$ is bounded for each $\nu$. We are interested in finding asymptotic critival values for the test statistic

$$
2 \Lambda_{N}(\hat{\nu})=\sup _{a \leq \nu \leq b} 2 \Lambda_{N}(\nu), \text { where } \hat{\nu} \text { is the MLE of } \nu
$$

We start with the second-order Taylor-Series expansion:

$$
2 \Lambda_{N}(\nu)=\left(\frac{T_{1}(\nu)}{K_{1}(\nu)}-\frac{T_{2}(\nu)}{K_{2}(\nu)}\right)^{2}\left(\frac{K_{1}(\nu) K_{2}(\nu)}{K_{1}+K_{2}}\right)\left(\frac{K_{1}+K_{2}}{T_{1}+T_{2}}\right)^{2}+R_{n}(\nu) .
$$

Proposition 1. For any $0<a<b<1$,

$$
\sup _{a \leq \nu \leq b}\left|R_{n}(\nu)\right| \rightarrow^{p} 0
$$

as $n \rightarrow \infty$, when $H_{0}$ is true.

Proof. See the proof of Proposition 1 in Kim, Woodroofe and Wu (2004).

We define $Z_{N}(\nu)$ as the following:

$$
Z_{N}(\nu)=\left(\frac{T_{1}(\nu)}{K_{1}(\nu)}-\frac{T_{2}(\nu)}{K_{2}(\nu)}\right)\left(\frac{K_{1}(\nu) K_{2}(\nu)}{K_{1}+K_{2}}\right)^{1 / 2}\left(\frac{K_{1}+K_{2}}{T_{1}+T_{2}}\right) .
$$

The square of the $Z_{N}(\nu)$ process will provide an approximation to the $2 \Lambda_{N}(\nu)$ process.

Theorem 2. Under $H_{0}, Z_{N}(\nu)$ converges weakly to the zero-mean, unit-variance, Gaussian process $Z(\nu)$, where

$$
\rho_{12}=\operatorname{Cov}\left(Z\left(\nu_{1}\right), Z\left(\nu_{2}\right)\right)=\left(\frac{P\left(x<\nu_{1}, \delta=1\right)}{P\left(x<\nu_{2}, \delta=1\right)} \times \frac{P\left(x \geq \nu_{2}, \delta=1\right)}{P\left(x \geq \nu_{1}, \delta=1\right)}\right)^{1 / 2} .
$$

and 


$$
\begin{aligned}
P(x<\nu, \delta & =1)=1-e^{-\frac{\theta}{k} \nu^{k}}-\frac{\theta}{k T} \int_{0}^{\nu^{k}} z^{1 / k} e^{-\frac{\theta}{k} z} d z \\
P(x \geq \nu, \delta & =1)=e^{-\frac{\theta}{k} \nu^{k}}-e^{-\frac{\theta}{k} T^{k}}-\frac{\theta}{k T} \int_{\nu^{k}}^{T^{k}} z^{1 / k} e^{-\frac{\theta}{k} z} d z \\
P(\delta=1) & =1-e^{-\frac{\theta}{k} T^{k}}-\frac{\theta}{k T} \int_{0}^{T^{k}} z^{1 / k} e^{-\frac{\theta}{k} z} d z .
\end{aligned}
$$

Proof. See Appendix.

We can see that $Z(\nu)$ is non-stationary, but we can transform $Z(\nu)$ into an Ornstein-Uhlenbeck process $W(t)$, which is stationary.

Corollary 3. For $Z(\nu)$ defined above, $g_{1}(\nu)=P(x<\nu, \delta=1)$, and $g_{2}(\nu)=$ $P(x \geq \nu, \delta=1)$

$$
W(t)=Z\left(g_{2}^{-1}\left(\frac{g_{2}(0) e^{t}}{1+e^{t}}\right)\right), t \in(-\infty, \infty)
$$

is an Ornstein-Uhlenbeck process.

Proof. Both $g_{1}$ and $g_{2}$ are positive, $g_{1}$ is increasing, and $g_{2}$ is decreasing on $\nu \in$ $[0, T]$. Furthermore, $g_{1}(\nu)=g_{2}(0)-g_{2}(\nu)$. We can use the following relationship,

$$
g_{2}(\nu)=g_{2}(0) \frac{e^{t}}{1+e^{t}}, t \in(-\infty, \infty)
$$

Then for $\nu_{1}<\nu_{2} \Leftrightarrow t_{1}>t_{2}$,

$$
\begin{aligned}
\rho_{12} & =\sqrt{\frac{P\left(x<\nu_{1}, \delta=1\right)}{P\left(x<\nu_{2}, \delta=1\right)} \times \frac{P\left(x \geq \nu_{2}, \delta=1\right)}{P\left(x \geq \nu_{1}, \delta=1\right)}} \\
& =\sqrt{\frac{e^{t_{2}}}{e^{t_{1}}}} \\
& =e^{-\frac{1}{2}\left|t_{1}-t_{2}\right|} .
\end{aligned}
$$

An approximation of the tail probability for the supremum of an OrnsteinUhlenbeck process can be derived:

\section{Corollary 4.}

$$
\begin{aligned}
P\left[\sup _{a \leq \nu \leq b} Z(\nu)^{2}>c^{2}\right] & =P\left[\sup _{a \leq \nu \leq b}|Z(\nu)|>c\right] \\
& =P\left[\sup _{a^{*} \leq t \leq b^{*}}|W(t)|>c\right] \\
& \approx\left(b^{*}-a^{*}\right) \frac{c}{\sqrt{2 \pi}} e^{-c^{2} / 2}
\end{aligned}
$$


where $a^{*}=\log \left[g_{2}(b) /\left(g_{2}(0)-g_{2}(b)\right)\right], b^{*}=\log \left[g_{2}(a) /\left(g_{2}(0)-g_{2}(a)\right)\right]$, and $c>0$ is large.

Proof. See Theorem 12.2.9 and Remark 12.2.10 in Leadbetter, Lindgren and Rootzén (1983).

3. Other forms of type I censoring: If we consider the same underlying hazard function (1.1), but assume a different form of type I censoring, we might still be able to establish convergence similar to that of Theorem 2. In the proof of weak convergence (See Appendix), the step that directly relies on censoring probabilities uses the equality

$$
\frac{1}{k} E\left(x^{k} \wedge \nu^{k}\right)=\frac{1}{\theta} P(X \leq \nu, \delta=1) .
$$

This should establish weak convergence, but might not lead to the same covariance structure as in Theorem 2 .

One example occurs when all subjects enter at time $\tau=0$ and the study ends at time $T$. This situation satisfies (3.1) and has an identifiable covariance structure. In this case, the covariance is of the same form as in Theorem 2, and the correlation $\rho_{12}$ now contains the following probabilities:

$$
\begin{aligned}
P(x<\nu, \delta & =1)=1-e^{-\frac{\theta}{k} \nu^{k}} \\
P(x \geq \nu, \delta & =1)=e^{-\frac{\theta}{k} \nu^{k}}-e^{-\frac{\theta}{k} T^{k}} \\
P(\delta=1) & =1-e^{-\frac{\theta}{k} T^{k}} .
\end{aligned}
$$

If we let $T \rightarrow \infty$, we also get a result for the case of no censoring.

4. Simulation Study: We turn to the Rayleigh case, with $k=2$. The hazard function is linear and at an unknown time, $\nu>0$, the slope of the hazard function changes:

$$
h(t)=\left\{\begin{array}{ll}
\theta_{1} t, & t<\nu \\
\theta_{2} t, & t \geq \nu
\end{array}\right\}, \theta_{1}>0, \theta_{2}>0, t \geq 0 .
$$

In order to get critical values for $2 \Lambda_{N}(\hat{\nu})$, we could simulate $\left\{x_{i}, \delta_{i}\right\}$ under the null hypothesis $\theta_{1}=\theta_{2}=\theta_{0}$ and search $2 \Lambda_{N}(\nu)$ over a fine grid of $\nu \in(a, b) \subset$ $(0, T)$ to find the maximum value. We would repeat this many times and record the sample quantiles. Alternatively, we could calculate critical values much more 
Table 4.1: Critical Values for $\sup Z(\nu)^{2}$

\begin{tabular}{|c|c|c|c|c|c|c|c|c|}
\hline \multicolumn{3}{|c|}{ Parameters } & \multicolumn{2}{c|}{ Simulated } & \multicolumn{3}{c|}{ O-U approx. } & Censoring \\
\hline$\theta$ & $\mathrm{T}$ & $\mathrm{a}$ & $\mathrm{b}$ & $95 \%$ & $99 \%$ & $95 \%$ & $99 \%$ & $\mathrm{P}(\delta=0)$ \\
\hline 0.25 & 1 & 0.2 & 0.8 & 9.21 & 12.69 & 9.32 & 12.86 & $96 \%$ \\
& & 0.3 & 0.7 & 8.20 & 11.64 & 8.16 & 11.74 & \\
& & 0.4 & 0.6 & 7.09 & 10.49 & 6.43 & 10.10 & \\
\hline 1 & 1 & 0.2 & 0.8 & 9.30 & 12.78 & 9.36 & 12.90 & $85.6 \%$ \\
& & 0.3 & 0.7 & 8.29 & 11.90 & 8.20 & 11.79 & \\
& & 0.4 & 0.6 & 7.04 & 10.48 & 6.47 & 10.14 & \\
\hline 4 & 1 & 0.2 & 0.8 & 9.44 & 12.97 & 9.56 & 13.10 & $59.8 \%$ \\
& & 0.3 & 0.7 & 8.42 & 11.83 & 8.43 & 12.00 & \\
& & 0.4 & 0.6 & 7.23 & 10.62 & 6.71 & 10.36 & \\
\hline
\end{tabular}

rapidly by using $Z(\nu)$ instead of $2 \Lambda_{N}(\nu)$. Computationally, the probabilities in $\rho_{12}$ are straight-forward to calculate with software by converting the integrals into cumulative distribution functions for gamma random variables. Another alternative is to use the approximation from Corollary 4. This last method is almost instantaneous. In the following simulations, we compare the properties of critical values generated by our two approximations in terms of type I error rates and power.

4.1 Critical Values: Under the null hypothesis, we simulate 50000 realizations of $Z(\nu)$ for different combinations of parameters, with the grid of $\nu$ over $10^{-4}$ increments (Table 4.1). Most of our data are censored. We use a simple non-linear solver to find the approximate critical values using Corollary 4. The two sets of critical values seem to match up well for the $[0.2,0.8]$ and $[0.3,0.7]$ intervals. The values from the approximation of the Ornstein-Uhlenbeck supremum seem too low for the $[0.4,0.6]$ intervals.

4.2 Critical Values for $\theta_{0}$ unknown: The $g_{2}$ function from Corollary 4 is a function of $\theta_{0}$, the slope under the null hypothesis. However, the critical values are well-behaved for the ranges of $\theta_{0}$ considered here. Numerically, the critical values appear to be monotonic with respect to $\theta_{0}$. We can demonstrate this by showing that correlations between $Z\left(\nu_{i}\right)$ and $Z\left(\nu_{j}\right)$ are decreasing with increasing $\theta$ (Figure 4.1 ) or by showing that $\left(b^{*}-a^{*}\right)$ is increasing with respect to $\theta$ (Figure 4.2). The first case implies that $\sup _{a \leq \nu \leq b} Z(\nu)^{2}$ increases with $\theta$, because a realization of the process becomes closer to a draw of independent $Z\left(\nu_{i}\right)$. The 
second case implies that our approximation from Corrollary 4 increases with $\theta$.

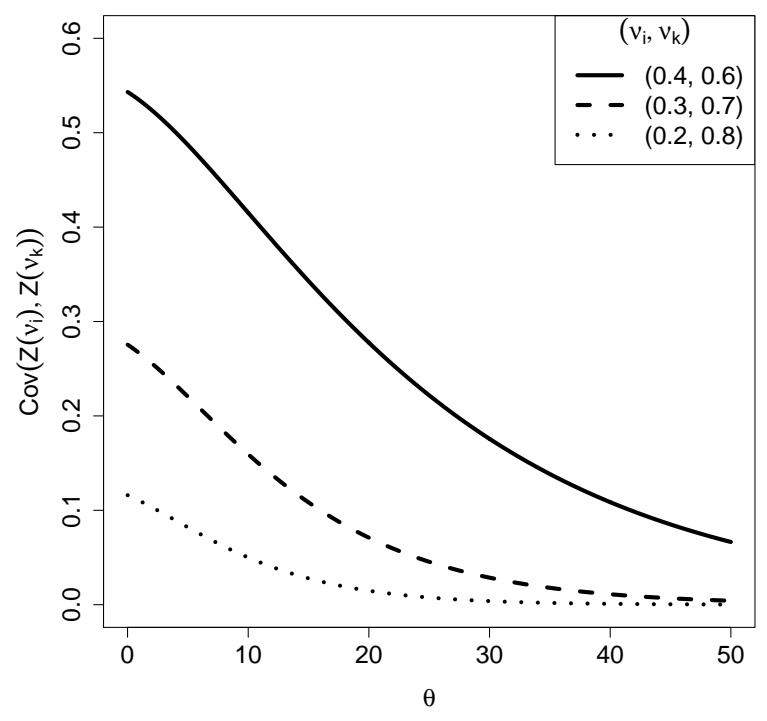

Figure 4.1: $\operatorname{Cov}\left(Z\left(\nu_{i}\right), Z\left(\nu_{j}\right)\right)$ decreases as $\theta$ increases

This suggests that it's possible to be conservative by choosing $\theta_{0}$ larger than some estimate $\hat{\theta}_{0}$. Alternatively, we propose estimating $\theta_{0}$ by using the observed proportion of censored observations as an estimate of $P(\delta=0)$ and solving for $\theta_{0}$. For the range of $\theta$ considered, this leads to accurate estimates of both $\theta_{0}$ and the corresponding critical values (Table 4.2). Furthermore, larger variability in $\hat{\theta}_{0}$ corresponds to smaller variability in estimates of the critical values. The larger variation for $\theta_{0}=4$ suggests that this approach is reasonable as long as the censoring proportion is moderate to large.

4.3 Empirical Error Probabilities: Under the null hypothesis, we simulate data from a Rayleigh distribution for $\theta \in\{0.25,1,4\}, T=1,(a, b) \in$ $\{(0.2,0.8),(0.3,0.7),(0.4,0.6)\}$, with the grid of $\nu$ over $10^{-4}$ increments. To simulate the Poisson process of entry into the study, we generate $N \sim \operatorname{Poisson}(\gamma)$ with $\gamma \in(50,100,200,500,1000)$. We then sample $Y_{i} \sim \operatorname{Rayleigh}(\theta)$ for $i \in(1, \ldots, N)$. Finally we sample the censoring times $\left(T-\tau_{i}\right) \sim \mathrm{U}(0, T)$. We then calculate $X_{i}$ and $\delta_{i}$ and form the log-likelihood ratio test statistic $2 \Lambda_{N}(\hat{\nu})$. We track the rate of exceeding the simulated critical values and the Ornstein-Uhlenbeck critical 

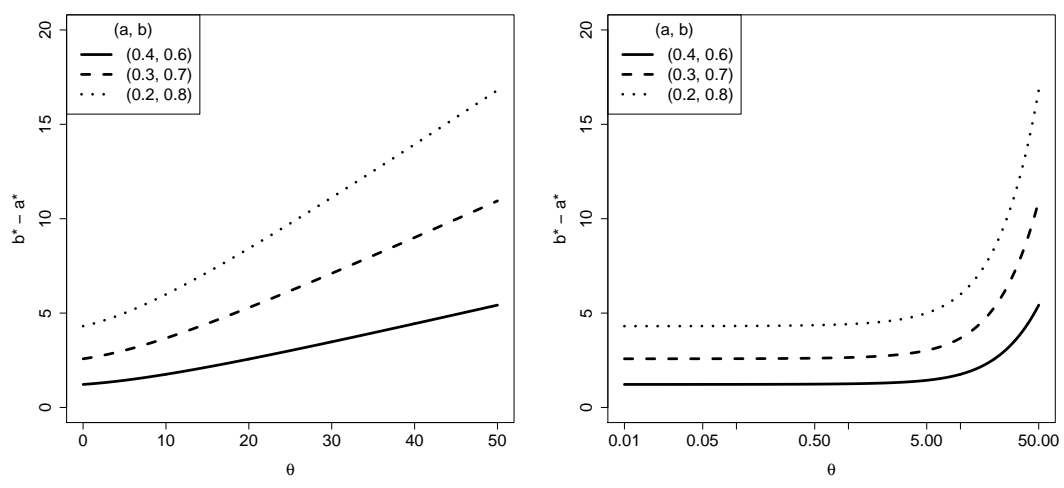

Figure 4.2: $\left(b^{*}-a^{*}\right)$ increases as $\theta$ increases

values (for both $\theta_{0}$ known and unknown) and compare these rates to the nominal type I error rates. From Table 4.3, the simulated critical values seem to attain the nominal type I error rates of $5 \%$ around $\gamma=100$ for $\theta=4, \gamma=500$ for $\theta=1$, and $\gamma=1000$ (or perhaps even 2000) for $\theta=0.25$. The Ornstein-Uhlenbeck critical values give results that are very similar. The exception is the $[0.4,0.6]$ case, for which the type I error rates are too large. Results for $1 \%$ rates are similar and thus not included.

4.4 Power: Under the alternative hypothesis, we simulate data using $\theta_{1}=$ $0.25,1,4$ and $\theta_{2} / \theta_{1}=(1 / 3,1 / 2.8, \ldots, 2.8,3.0)$ with $\gamma=500$. We then track the rate of exceeding the simulated critical values. We first consider a change at $\nu=0.5$. From Figure 4.3 we see that the censoring rate affects power. For $\theta_{1}=4$ the power is higher than for $\theta_{1}=1$ which is higher than that for $\theta_{1}=0.25$. The censoring rate is the lowest for $\theta_{1}=4$, so we would expect to see many more uncensored observations than for the other two settings. Figure 4.4 shows that all three methods for obtaining critical values (i.e. the simulated values and the Ornstein-Uhlenbeck approximation with and without $\theta_{0}$ known) lead to essentially the same power for moderate to wide intervals.

Within values of $\theta_{1}$ (Figure 4.5), the narrowest interval $[0.4,0.6]$ provides the highest power, while the widest interval $[0.2,0.8]$ has the lowest power for a change at 0.5 . For a change outside of $[0.4,0.6]$, this is no longer true. Figure 4.6 shows that the interval $[0.3,0.7]$ has the best power for $\nu=0.65$, and $[0.4,0.6]$ 
Table 4.2: Critical Values (5\%) for the O-U approx. with $\theta_{0}$ unknown

\begin{tabular}{|c|c|c|c|c|c|c|}
\hline \multicolumn{2}{|c|}{} & \multicolumn{5}{|c|}{ Simulations Estimating $\theta_{0}(1000$ each $)$} \\
\hline \multicolumn{2}{|c|}{ True Values } & Mean & SD & $Q_{5}$ & $Q_{50}$ & $Q_{95}$ \\
\hline$\theta_{0}$ & 0.250 & 0.249 & 0.057 & 0.163 & 0.244 & 0.347 \\
\hline $\mathrm{O}-\mathrm{U}[0.4,0.6]$ & 6.425 & 6.425 & 0.004 & 6.420 & 6.425 & 6.431 \\
\hline $\mathrm{O}-\mathrm{U}[0.3,0.7]$ & 8.159 & 8.159 & 0.003 & 8.154 & 8.159 & 8.165 \\
\hline $\mathrm{O}-\mathrm{U}[0.2,0.8]$ & 9.318 & 9.318 & 0.003 & 9.313 & 9.317 & 9.323 \\
\hline$\theta_{0}$ & 1.000 & 0.998 & 0.126 & 0.795 & 0.995 & 1.208 \\
\hline $\mathrm{O}-\mathrm{U}[0.4,0.6]$ & 6.474 & 6.474 & 0.009 & 6.460 & 6.473 & 6.488 \\
\hline $\mathrm{O}-\mathrm{U}[0.3,0.7]$ & 8.205 & 8.205 & 0.008 & 8.192 & 8.205 & 8.218 \\
\hline $\mathrm{O}-\mathrm{U}[0.2,0.8]$ & 9.360 & 9.360 & 0.007 & 9.348 & 9.360 & 9.372 \\
\hline$\theta_{0}$ & 4.000 & 4.027 & 0.372 & 3.459 & 3.992 & 4.674 \\
\hline $\mathrm{O}-\mathrm{U}[0.4,0.6]$ & 6.709 & 6.712 & 0.032 & 6.663 & 6.709 & 6.768 \\
\hline $\mathrm{O}-\mathrm{U}[0.3,0.7]$ & 8.425 & 8.428 & 0.030 & 8.382 & 8.425 & 8.481 \\
\hline $\mathrm{O}-\mathrm{U}[0.2,0.8]$ & 9.563 & 9.565 & 0.028 & 9.523 & 9.562 & 9.614 \\
\hline
\end{tabular}

Table 4.3: Empirical Type I errors using 5\% Simulated Critical Values

\begin{tabular}{|c|c|c|c|c|c|c|c|c|}
\hline \multicolumn{4}{|c|}{ Parameters } & \multicolumn{5}{c|}{ Expected Sample Size $(\mathrm{se} \approx 0.0020)$} \\
\hline$\theta$ & $\mathrm{T}$ & $\mathrm{a}$ & $\mathrm{b}$ & 50 & 100 & 200 & 500 & 1000 \\
\hline 0.25 & 1 & 0.2 & 0.8 & 0.0010 & 0.0055 & 0.0159 & 0.0333 & 0.0426 \\
& & 0.3 & 0.7 & 0.0009 & 0.0046 & 0.0188 & 0.0410 & 0.0481 \\
& & 0.4 & 0.6 & 0.0001 & 0.0025 & 0.0228 & 0.0486 & 0.0460 \\
\hline 1 & 1 & 0.2 & 0.8 & 0.0164 & 0.0246 & 0.0354 & 0.0472 & 0.0503 \\
& & 0.3 & 0.7 & 0.0155 & 0.0372 & 0.0484 & 0.0477 & 0.0475 \\
& & 0.4 & 0.6 & 0.0173 & 0.0470 & 0.0500 & 0.0486 & 0.0492 \\
\hline 4 & 1 & 0.2 & 0.8 & 0.0628 & 0.0445 & 0.0482 & 0.0485 & 0.0468 \\
& & 0.3 & 0.7 & 0.0512 & 0.0483 & 0.0490 & 0.0501 & 0.0506 \\
& & 0.4 & 0.6 & 0.0449 & 0.0487 & 0.0493 & 0.0463 & 0.0514 \\
\hline
\end{tabular}


has the worst. Results are similar for $\nu=0.35$ (Figure not shown). This suggests that while the narrowest interval should have the best power, a poor choice of interval will cause a narrow interval to be less powerful than a wider one. Results for $\alpha=0.01$ are similar (Figure not included).

From Figure 4.3, it is clear that these power curves are not symmetric. The test has greater power detecting a change when $\log \left(\theta_{2} / \theta_{1}\right)$ is positive. Kim, Woodroofe, and $\mathrm{Wu}$ (2004) use Hellinger distance to justify this for the exponential case. We explain using censoring rates. For a given $\theta_{1}$ under the alternative hypothesis, the hazard function, $h(t)$ has the following property: $h\left(t ;\left(\theta_{2} / \theta_{1}\right)>1\right) \geq h\left(t ;\left(\theta_{2} / \theta_{1}\right) \leq 1\right)$ for all $t$. Then the cumulative distribution function also has this property: $F\left(t ;\left(\theta_{2} / \theta_{1}\right)>1\right) \geq F\left(t ;\left(\theta_{2} / \theta_{1}\right) \leq 1\right)$ for all $t$. Therefore we expect to see more uncensored events when $\log \left(\theta_{2} / \theta_{1}\right)>0$, assuming the same censoring conditions. As demonstrated above, power is influenced by the number of uncensored observations. When $\log \left(\theta_{2} / \theta_{1}\right)>0$, we expect to have more uncensored observations than when $\log \left(\theta_{2} / \theta_{1}\right) \leq 0$. So when $\left|\log \left(\theta_{2} / \theta_{1}\right)\right|=r>0$, we should have greater power for the right tail than for the left.

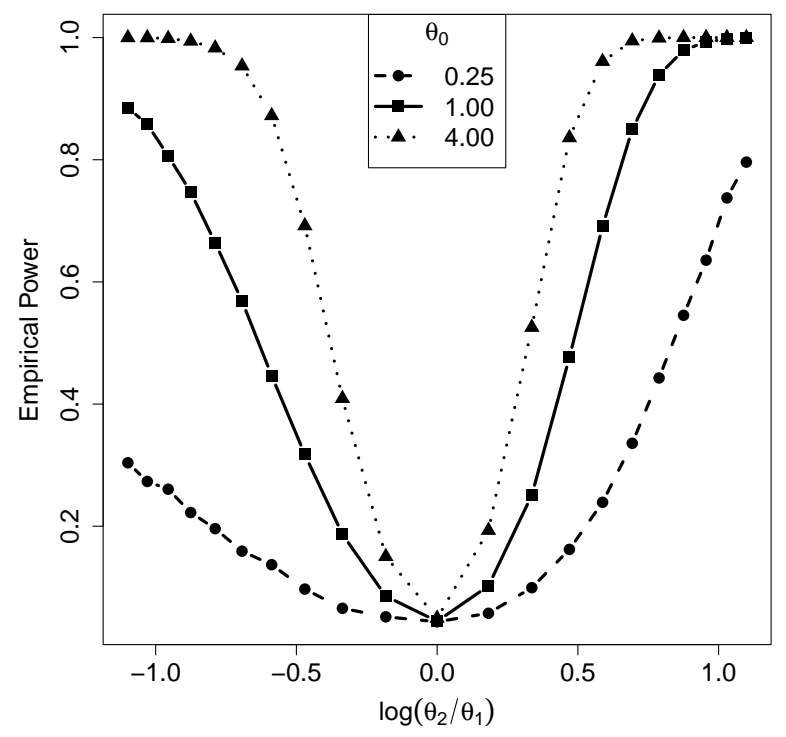

Figure 4.3: Empirical power at the $5 \%$ level for $\gamma=500$ and $[a, b]=[0.3,0.7]$ 

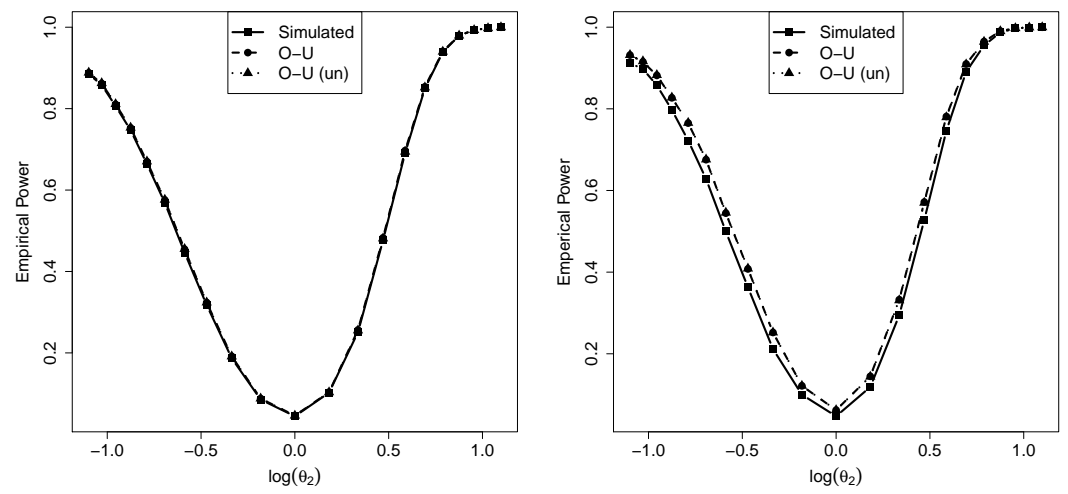

Figure 4.4: Empirical Power for $\theta_{1}=1, \gamma=500$, and $\nu=0.5$. Comparing Simulated and Ornstein-Uhlenbeck (O-U) $5 \%$ critical values from Tables 4.1 and 4.2. Left to right $[0.3, .07]$ and $[0.4,0.6]$

5. Clinical Study Example: We now apply our testing procedure to the results of a placebo controlled trial of gamma interferon in the treatment of chronic granulomatous disease (Fleming and Garrington, 1991). This is a hereditary disease of the immune system that can lead to serious infections. The example data are the times to first infection since the start of treatment. These times appear to follow a Rayleigh distribution subject to staggered entry and right censoring. Times have been rescaled by dividing by the length of the study $(\mathrm{T}=$ 321 days) to have scaling consistent with our simulations $(\mathrm{T}=1)$. Weibull plots of the placebo and treatment groups support that $k=2$ is reasonable (Figure 5.7). We can see this by noting that for later events the treatment and placebo groups have curves that are roughly parallel with slope of about 2 . The maximum likelihood estimate of $k$ for the treatment group is 1.80. If we split the placebo group into two groups (before and after 0.10), we get estimates of 2.19 and 1.96 for the shape parameter. Together this all supports the assumption that $k=2$ is reasonable. The early failures for the placebo group suggest a potential shift in the scale parameter for the placebo group but no shift for the treatment group.

We now test the placebo data to see if the alternative (4.1) is reasonable. There are 53 patients in the placebo group and 20 observed events for a censoring proportion of around $60 \%$. From Figure 5.7, it appears that a change might occur early on, so we choose a wide interval $\nu \in[0.05,0.95]$. We calculate critical 


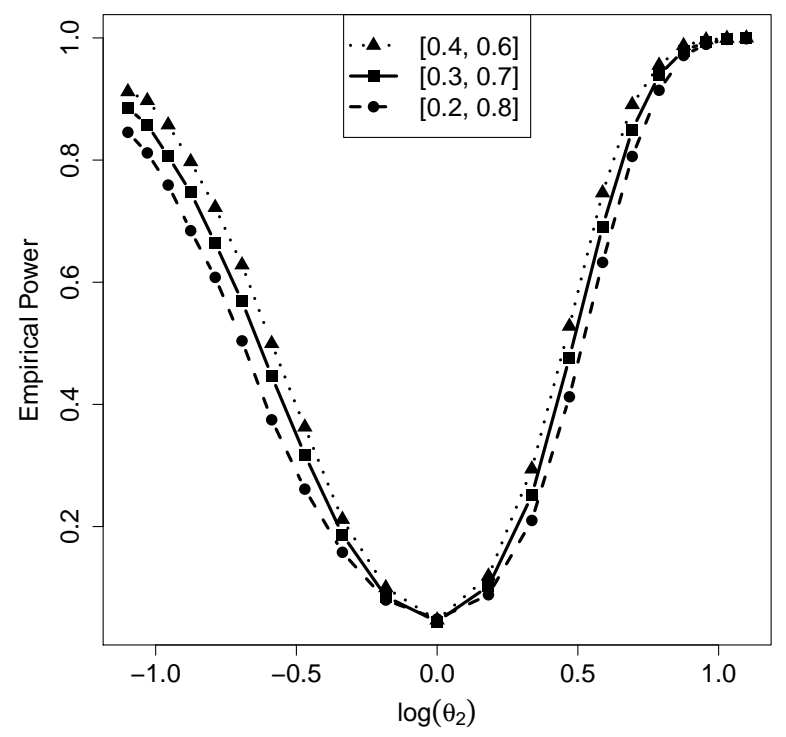

Figure 4.5: Empirical power at the $5 \%$ level with $\theta_{1}=1, \gamma=500$, and $\nu=0.5$

values using the method described in Section 4.2. The plug-in estimate $\hat{\theta}_{0}$ is 3.60 , and the asymptotic $5 \%$ and $1 \%$ critical values are 11.30 and 14.79 respectively. The likelihood ratio test statistic is 37.79 (maximized over a $10^{-4}$ grid of $\nu$ ). We therefore reject the null hypothesis $\theta_{1}=\theta_{2}=\theta_{0}$ in favor of the alternative $\theta_{1} \neq \theta_{2}$. The corresponding point of maximization is $\hat{\nu}=0.0717$, which translates to 23.02 days on our original scale. The conditional MLE's are $\hat{\theta}_{1}(\hat{\nu})=64.69$ and $\hat{\theta}_{2}(\hat{\nu})=1.78$. We can also test for a change in the treatment group. There are 55 patients receiving the treatment and only 7 uncensored observations. The likelihood ratio test statistic is 1.63 . The test statistic is so small that we would not expect to reject the null hypothesis. These results suggest that the treatment may remove the early failure structure that is present under placebo conditions. 6. Conclusions: We have established a generalization of the procedure for testing abrupt changes under staggered-entry established by Kim, Woodroofe and $\mathrm{Wu}(2004)$ by extension to hazard functions of the Weibull form. We have also evaluated one method of implementing this test by estimating $\theta_{0}$ to calculate critical values, and demonstrated its effectiveness through both simulation and a 


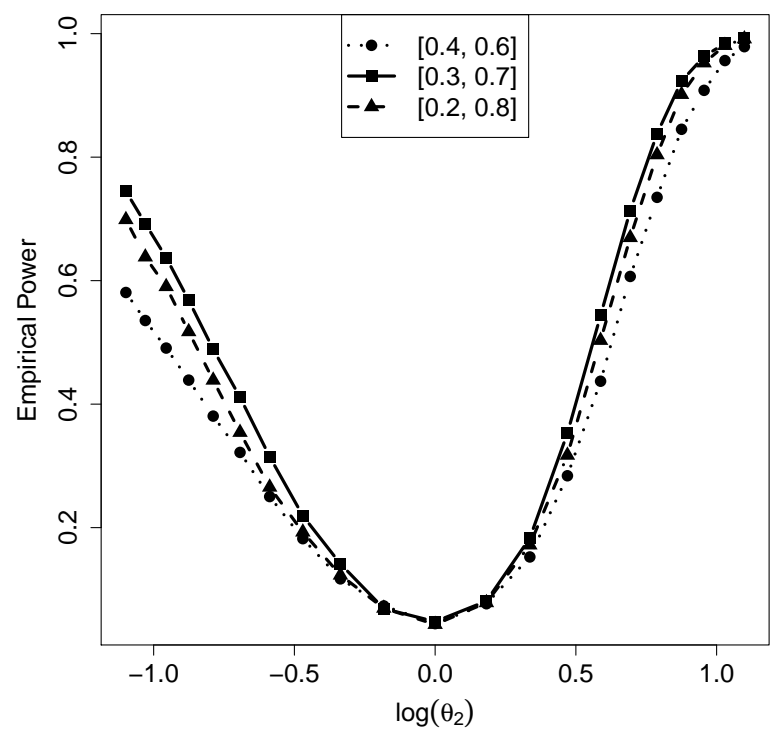

Figure 4.6: Empirical Power at the 5\% level for $\theta_{1}=1, \gamma=500$, and $\nu=0.65$

clinical study example. The convergence rate of the test is related to the number of uncensored observations, suggesting that around 50 uncensored observations provides adequate convergence. Furthermore, power appears to increase as the proportion of uncensored observations increases. Extensions of this work might include the use of covariates and exploring other forms of censoring.

\section{Acknowledgments}

The first author is sponsored through the Institute for Critical Technology and Applied Science (ICTAS) Doctoral Scholars Program at Virginia Tech. The work of the second author has been funded as part of the National Science Foundation Biocomplexity of Coupled Human and Natural Systems Program, Award No. BCS-0709671

7. Appendix: We prove weak convergence and find the covariance for the Gaussian process $Z(\nu)$ in a manner similar to Kim, Woodroofe and Wu (2004).

Proof of Weak Convergence. We show that $Z_{N}(\nu)$ converges weakly to a Gaussian process by expressing it in terms of empirical and sub-empirical processes, $\mathbb{F}_{N}(x)$ and $\tilde{\mathbb{F}}_{N}(x)$, defined by 


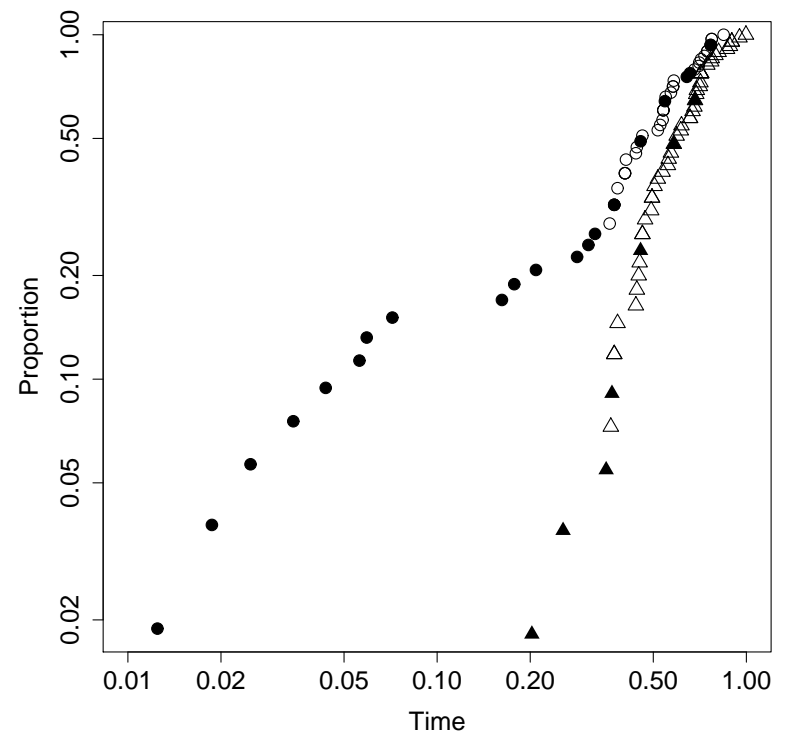

Figure 5.7: Time to first infection vs Proportion of events. Placebo (circle), treatment (triangle), and censored (outline).

$$
\begin{aligned}
& \mathbb{F}_{N}(x)=\sqrt{N}\left[F_{N}(x)-F(x)\right] \\
& \tilde{\mathbb{F}}_{N}(x)=\sqrt{N}\left[\tilde{F}_{N}(x)-\tilde{F}(x)\right]
\end{aligned}
$$

where $F_{N}(x)$ and $\tilde{F}_{N}(x)$ are the empirical and subempirical cumulative distribution functions

$$
\begin{aligned}
& F_{N}(x)=\frac{1}{N} \sum_{i=1}^{N} 1_{\left\{X_{i} \leq x\right\}} \\
& \tilde{F}_{N}(x)=\frac{1}{N} \sum_{i=1}^{N} 1_{\left\{X_{i} \leq x\right\}} \delta_{i}
\end{aligned}
$$

and $F(x)$ and $\tilde{F}(x)$ are their asymptotic distributions under the null hypothesis

$$
\begin{aligned}
& F(x)=P\left(X_{i} \leq x\right)=1-\frac{1}{T}(T-x) e^{-\left(\theta x^{k} / k\right)} \\
& \tilde{F}(x)=P\left(X_{i} \leq x, \delta_{i}=1\right)=1-e^{-\left(\theta x^{k} / k\right)}-\frac{\theta}{k T} \int_{0}^{x^{k}} z^{1 / k} e^{-(\theta z / k)} d z .
\end{aligned}
$$

We note that (expressing $T_{j}(\nu)$ as $T_{j}$ and $K_{j}(\nu)$ as $K_{j}$ for ease of notation)

$$
\frac{T_{1}}{K_{1}}-\frac{T_{2}}{K_{2}}=\frac{1}{K_{1} K_{2}}\left[K_{2}\left(T_{1}-\frac{K_{1}}{\theta}\right)-K_{1}\left(T_{2}-\frac{K_{2}}{\theta}\right)\right]
$$


We note further that $K_{1} / N=\tilde{F}_{N}(\nu)$ and $K_{2} / N=\tilde{F}_{N}(T)-\tilde{F}_{N}(\nu)$.

Next we express $T_{1} / N$ in terms of the empirical distribution function.

$$
\begin{aligned}
\frac{T_{1}}{N} & =\frac{1}{k N} \sum_{i=1}^{N}\left(\int_{0}^{\nu^{k}} 1_{\left\{x_{i}^{k}>t^{k}\right\}} d\left(t^{k}\right)\right) \\
& =\frac{1}{k} \int_{0}^{\nu^{k}}\left[1-F_{N}(t)\right] d\left(t^{k}\right) .
\end{aligned}
$$

Similarly, $T_{2} / N=k^{-1} \int_{\nu^{k}}^{T^{k}}\left[1-F_{N}(t)\right] d\left(t^{k}\right)$ and $k^{-1} E\left(x^{k} \wedge \nu^{k}\right)=k^{-1} \int_{0}^{\nu^{k}}[1-F(t)] d\left(t^{k}\right)$.

Through integration, we see that $k^{-1} E\left(x^{k} \wedge \nu^{k}\right)=\theta^{-1} P(X \leq \nu, \delta=1)=$ $\theta^{-1} \tilde{F}(\nu)$.

We can express $T_{1}-K_{1} \theta^{-1}$ and $T_{2}-K_{2} \theta^{-1}$ in terms of the empirical and subempirical processes:

$$
\begin{aligned}
T_{1}-\frac{K_{1}}{\theta} & =-\frac{\sqrt{N}}{k} \int_{0}^{\nu^{k}} \mathbb{F}_{N}(x) d\left(x^{k}\right)-\frac{\sqrt{N}}{\theta} \tilde{\mathbb{F}}_{N}(\nu) \\
& =-\sqrt{N} \int_{0}^{\nu} x^{k-1} \mathbb{F}_{N}(x) d(x)-\frac{\sqrt{N}}{\theta} \tilde{\mathbb{F}}_{N}(\nu), \\
T_{2}-\frac{K_{2}}{\theta} & =-\frac{\sqrt{N}}{k} \int_{\nu^{k}}^{T^{k}} \mathbb{F}_{N}(x) d\left(x^{k}\right)-\frac{\sqrt{N}}{\theta}\left[\tilde{\mathbb{F}}_{N}(T)-\tilde{\mathbb{F}}_{N}(\nu)\right] \\
& =-\sqrt{N} \int_{\nu}^{T} x^{k-1} \mathbb{F}_{N}(x) d(x)-\frac{\sqrt{N}}{\theta}\left[\tilde{\mathbb{F}}_{N}(T)-\tilde{\mathbb{F}}_{N}(\nu)\right] .
\end{aligned}
$$

Furthermore, $Z_{N}(\nu)$ can be expressed as

$$
\begin{aligned}
Z_{N}(\nu) & =C_{N}\left(\frac{K_{2}}{N}, \frac{K_{1}}{N}\right) \frac{1}{\sqrt{N}}\left(T_{1}-\frac{K_{1}}{\theta}, T_{2}-\frac{K_{2}}{\theta}\right)^{t} \\
& =C_{N}\left(\frac{K_{2}}{N}, \frac{K_{1}}{N}\right) \phi\left(\mathbb{F}_{N}, \tilde{\mathbb{F}}_{N}\right),
\end{aligned}
$$

where

$$
C_{N}=\left(\frac{K_{1}+K_{2}}{K_{1} K_{2}}\right)^{1 / 2} \frac{N^{3 / 2}}{T_{1}+T_{2}}
$$

and $\phi: D[0, T] \times D[0, T] \rightarrow D[0, T] \times D[0, T]$ such that

$$
\phi(g, h)(\nu)=\left(-\int_{0}^{\nu} x^{k-1} g(x) d x-\frac{1}{\theta} h(\nu),-\int_{\nu}^{T} x^{k-1} g(x) d x-\frac{1}{\theta}[h(T)-h(\nu)]\right)^{t}
$$

We observe that $\phi$ is continuous at every $(g, h)$ for $g$ and $h$ in $D[0, T]$. Then all sequences $g_{N}(x)$ and $h_{N}(x)$ that converge to $g(x)$ and $h(x)$ respectively, converge uniformly on $x \in[0, T]$. It follows that $\phi\left(g_{N}, h_{N}\right)(\nu)$ must also converge uniformly to $\phi(g, h)(\nu)$ for $x \in[0, T]$.

We establish weak convergence by using Theorem 3 from Breslow and Crow- 
ley (1974), in which $\left\{\mathbb{F}_{N}, \tilde{\mathbb{F}}_{N}\right\}$ converge weakly to a bivariate process $\{G, \tilde{G}\}$. Kim, Woodroofe, and $\mathrm{Wu}(2004)$ note that $G$ has the same distribution as $\mathbb{B}(F)$ and $\tilde{G}$ has the same distribution as $\tilde{\mathbb{B}}(F)$, where $\mathbb{B}$ is a Brownian Bridge. This result, along with the continuity of $\phi$ implies that $Z_{N}(\nu)$ converges weakly to $Z(\nu)$, where

$$
Z(\nu)=C(\nu)[\tilde{F}(T)-\tilde{F}(\nu),-\tilde{F}(\nu)] \phi[\mathbb{B}(F), \mathbb{B}(\tilde{F})](\nu),
$$

with

$$
C(\nu)=\frac{k}{E\left(X^{k}\right)}\left(\frac{\tilde{F}(T)}{\tilde{F}(\nu)(\tilde{F}(T)-\tilde{F}(\nu))}\right)^{1 / 2} .
$$

Derivation of Covariance. To find $\operatorname{Cov}\left(Z\left(\nu_{1}\right), Z\left(\nu_{2}\right)\right)$, we first let $T_{1}-K_{1} \theta^{-1}=$ $\sum_{i=1}^{N} X_{i}^{\prime}(\nu)$ and $T_{2}-K_{2} \theta^{-1}=\sum_{i=1}^{N} X_{i}^{\prime \prime}(\nu)$, with

$$
\begin{aligned}
& X_{i}^{\prime}(\nu)=\frac{1}{k}\left(x_{i}^{k} \wedge \nu^{k}\right)-\frac{1}{\theta} 1_{\left\{x_{i}<\nu, \delta_{i}=1\right\}}, \\
& X_{i}^{\prime \prime}(\nu)=\frac{1}{k}\left(x_{i}^{k}-\nu^{k}\right)_{+}-\frac{1}{\theta} 1_{\left\{x_{i} \geq \nu, \delta_{i}=1\right\}}
\end{aligned}
$$

By the central limit theorem, for a given $\nu, \sqrt{N}\left(\sum X_{i}^{\prime}(\nu), \sum X_{i}^{\prime \prime}(\nu)\right)$ converges in distribution to the bivariate normal random variable $\left(Z_{1}(\nu), Z_{2}(\nu)\right)$ with mean vector $E\left(X^{\prime}, X^{\prime \prime}\right)$ and covariance $\operatorname{Cov}\left(X^{\prime}, X^{\prime \prime}\right)$. We can show $E\left(X^{\prime}(\nu)\right)=E\left(X^{\prime \prime}(\nu)\right)=$ 0 , and $\operatorname{Cov}\left(X^{\prime}(\nu), X^{\prime \prime}(\nu)\right)=0$.

Integration by parts and the transformation $z=x^{k}$ yield, for $\nu_{1} \leq \nu_{2}$,

$$
\begin{aligned}
\operatorname{Cov}\left(X^{\prime}\left(\nu_{1}\right), X^{\prime}\left(\nu_{2}\right)\right) & =\operatorname{Var}\left(X^{\prime}\left(\nu_{1}\right)\right)=\frac{1}{\theta^{2}} P\left(x<\nu_{1}, \delta=1\right)=\sigma_{1}^{2} \\
\operatorname{Cov}\left(X^{\prime \prime}\left(\nu_{1}\right), X^{\prime \prime}\left(\nu_{2}\right)\right) & =\operatorname{Var}\left(X^{\prime \prime}\left(\nu_{2}\right)\right)=\frac{1}{\theta^{2}} P\left(x \geq \nu_{2}, \delta=1\right)=\sigma_{4}^{2} \\
\operatorname{Cov}\left(X^{\prime}\left(\nu_{1}\right), X^{\prime \prime}\left(\nu_{2}\right)\right) & =0 \\
\operatorname{Cov}\left(X^{\prime}\left(\nu_{2}\right), X^{\prime \prime}\left(\nu_{1}\right)\right) & =\frac{1}{\theta^{2}}\left[P\left(x<\nu_{2}, \delta=1\right)-P\left(x<\nu_{1}, \delta=1\right)\right] \\
& =\left(\sigma_{2}^{2}-\sigma_{4}^{2}\right)=\left(\sigma_{3}^{2}-\sigma_{1}^{2}\right) .
\end{aligned}
$$


Let $\Sigma=\operatorname{Cov}\left(X^{\prime}\left(\nu_{1}\right), X^{\prime \prime}\left(\nu_{1}\right), X^{\prime}\left(\nu_{2}\right), X^{\prime \prime}\left(\nu_{2}\right)\right)$. Then

$$
\Sigma=\left(\begin{array}{cccc}
\sigma_{1}^{2} & 0 & \sigma_{1}^{2} & 0 \\
0 & \sigma_{2}^{2} & \left(\sigma_{2}^{2}-\sigma_{4}^{2}\right) & \sigma_{4}^{2} \\
\sigma_{1}^{2} & \left(\sigma_{3}^{2}-\sigma_{1}^{2}\right) & \sigma_{3}^{2} & 0 \\
0 & \sigma_{4}^{2} & 0 & \sigma_{4}^{2}
\end{array}\right) .
$$

We note that the covariance structure is based on the probability that an uncensored observation occurs before or after $\nu$, scaled by $\theta^{-2}$.

Now we can write

$$
\left[\begin{array}{c}
Z_{N}\left(\nu_{1}\right) \\
Z_{N}\left(\nu_{2}\right)
\end{array}\right]=A_{N}\left[\begin{array}{c}
\frac{1}{\sqrt{N}} \sum_{i=1}^{N} X_{i}^{\prime}\left(\nu_{1}\right) \\
\frac{1}{\sqrt{N}} \sum_{i=1}^{N} X_{i}^{\prime \prime}\left(\nu_{1}\right) \\
\frac{1}{\sqrt{N}} \sum_{i=1}^{N} X_{i}^{\prime}\left(\nu_{2}\right) \\
\frac{1}{\sqrt{N}} \sum_{i=1}^{N} X_{i}^{\prime \prime}\left(\nu_{2}\right)
\end{array}\right] \rightarrow^{d} A\left[\begin{array}{c}
Z_{1}\left(\nu_{1}\right) \\
Z_{2}\left(\nu_{1}\right) \\
Z_{1}\left(\nu_{2}\right) \\
Z_{2}\left(\nu_{2}\right)
\end{array}\right]=\left[\begin{array}{c}
Z\left(\nu_{1}\right) \\
Z\left(\nu_{2}\right)
\end{array}\right]
$$

where

$$
\begin{aligned}
A_{N} & =\left[\begin{array}{cccc}
a_{1 N}\left(\nu_{1}\right) & a_{2 N}\left(\nu_{1}\right) & 0 & 0 \\
0 & 0 & a_{1 N}\left(\nu_{2}\right) & a_{2 N}\left(\nu_{2}\right)
\end{array}\right], \\
A & =\left[\begin{array}{cccc}
a_{1}\left(\nu_{1}\right) & a_{2}\left(\nu_{1}\right) & 0 & 0 \\
0 & 0 & a_{1}\left(\nu_{2}\right) & a_{2}\left(\nu_{2}\right)
\end{array}\right]
\end{aligned}
$$

$\left(a_{1 N}(\nu), a_{2 N}(\nu)\right)=C_{N}(\nu)\left(K_{2} N^{-1}, K_{1} N^{-1}\right)$, and $\left(a_{1}(\nu), a_{2}(\nu)\right)=C(\nu)[\tilde{F}(T)-$ $\tilde{F}(\nu),-\tilde{F}(\nu)]$ from $(7.1)$ and $(7.2)$.

Then $\operatorname{Cov}\left(Z\left(\nu_{1}\right), Z\left(\nu_{2}\right)\right)=A \Sigma A^{t}=\left[\begin{array}{cc}1 & \rho_{12} \\ \rho_{21} & 1\end{array}\right]$.

\section{References}

Breslow, N. and Crowley, J. (1974). A large sample study of the life table and product limit estimates under random censorship. Ann Stat, 2, 437-453.

Davies, R. B. (1977). Hypothesis testing when a nuisance parameter is present only under the alternative. Biometrika, 64, 247-254.

Dupuy, J.-F. (2009). Detecting change in a hazard regression model with rightcensoring. Journal of Statistical Planning and Inference, 139, 1578-1586. 
Fleming, T. R. and Garrington, D. P. (1991). Counting Processes and Survival Analysis. John Wiley \& Sons.

Goodman, M. S., Li, Y. and Tiwari, R. C. (2006). Survival analysis with change point hazard functions. Harvard University Biostatistics Working Paper Series, 40.

Kim, D.-Y., Woodroofe, M. and Wu, Y. (2004). Testing for a change in the hazard rate with staggered entry. Commun Stat Theory, 33, 2041-2058.

Leadbetter, M. R., Lindgren, G. and Rootzén, H. (1983). Extremes and related properties of random sequences and processes. Springer, New York.

Loader, C. R. (1991). Inference for a hazard rate change point. Biometrika, 78 749-757.

Luo, X., Turnbull, B. W. and Clark, L. C. (1997). Likelihood ratio tests for a changepoint with survival data. Biometrika, 84 555-565.

Matthews, D. E. and Farewell, V. T. (1982). On testing for a constant hazard against a change-point alternative. Biometrics, 38 463-468.

Matthews, D. E. and Farewell, V. T. (1985). On a singularity in the likelihood for a change-point hazard rate model. Biometrika, 72703-704.

Matthews, D. E., Farewell, V. T. and Pyke, R. (1985). Asymptotic scorestatistic processes and tests for constant hazard against a change-point alternative. Ann Stat, 13 583-591.

Qin, J. and Sun, J. (1997). Statistical analysis of right-censored failure-time data with partially specified hazard rates. Can J Stat, 25 325-336.

Worsley, K. T. (1988). Exact percentage points of the likelihood-ratio test for a change-point hazard-rate model. Biometrics, 44 259-263. 